\title{
Can positivity be counterproductive when suffering domestic abuse?: A narrative review
}

\author{
Ellen Sinclair · Rona Hart · Tim Lomas
}

\begin{abstract}
Positive Psychology has been criticized for making people feel pressured to remain positive irrespective of circumstances. This narrative review specifically investigates the relationship between positive attitudes and denial in the context of domestic abuse, in order to examine whether there is research evidence to support the critique on the adverse upshots of positivity. The search yielded 29 studies. Overall the literature suggests that misdirected or overgeneralized positivity exacerbates harm and abuse: an optimistic bias can put victims in danger; empathy, hope, acceptance and resilience are associated with refraining to leave abusive relationships; and forgiveness increases the likelihood of further transgressions. We therefore argue that scholars and practitioners need demonstrate care in promoting positivity, since it can be detrimental within a toxic context.
\end{abstract}

Keywords: Domestic abuse, positivity, denial, narrative review

\section{Introduction}

Can a positive outlook promote denial or inaction in the context of hardship or abuse? The objective of this narrative review is to explore the empirical literature and inquire whether there is research evidence to suggest a that a positive outlook may lead to denial or inaction in the context of domestic abuse (DA). This review therefore asks whether a positive outlook (defined as encompassing constructs such as optimism, hope, gratitude, forgiveness, resilience, self-regulation, acceptance or empathy) can impede individuals from determining whether they are experiencing DA, and if this diminishes victims' capacity to engage appropriate coping strategies, or impacts their tendency to remain or leave the abusive relationship. This will raise a wider question about the risks entailed in unconditional positive orientation when it occurs within the context of hardship or abuse.

\section{The Risks of Positivity}

The positivity movement, advanced by the self-help industry since the early $20^{\text {th }}$ Century, has been growing in popularity. These ideas became an area of academic interest in the late nineties with the emergence of Positive Psychology (PP) (Seligman \& Csikszentmihalyi, 2000). One of the cornerstones of the positivity movement, which is well established in PP, is that psychological wellbeing is largely within an individual's control. Lyubomirsky $(2001,2013)$ suggests that happiness (subjective wellbeing) is on average only ten percent dependent on external 
circumstances, with a much larger percentage within one's own power to change by altering their thinking and behavioral patterns.

These ideas are attractive since they empower people to create their best possible experience of life. However, they also place pressure on individuals by implying that they are responsible for their own happiness; if their experience of life depends on their perception of the situation they are in, rather than an outcome of particular circumstances, it follows that if they are not happy, then the responsibility or 'blame' lies with them. Bohart and Greening (2001) warn against the misuse of PP 'to blame the victim for not having the proper optimistic attitude to achieve self-improvement in the face of massive social oppression' (p. 81). Coyne and Tennen (2010) voice a concern about the pressure that is generated when the media broadcasts farfetched claims about the power of positivity (such as its impact on illness recovery or longevity), which could be particularly harmful in the context of terminal illness (Coyne, Tennen \& Ranchor, 2010). Likewise, Held (2002) describes the pressure to be positive or happy as a distinctive cultural feature in North America. In acknowledging the influence of culture, she labels it as the 'tyranny of positivity'. She also observes that the pressure to be happy and optimistic under challenging circumstances can in effect diminish happiness. It can also drive people to ignore or deny negative emotions such as sadness or anger therefore hampering their capacity to evaluate life circumstances realistically (Held, 2001). While we refrain from this type of hard-edged judgement, we would concur with the view that there are certainly pressures towards being positive in many Western societies (and beyond), and that these have the potential to place burdens on people in the ways outlined above.

However, this critique of positive psychology is rarely backed by research evidence that can demonstrate the dangers of positivity in particular circumstances. This narrative review aims to address this gap in the literature by investigating the correlation between a positive attitude and denial or inaction in the context of relational hardship, specifically DA.

\section{The Context: Domestic Abuse}

Close relationships are one of the most significant factors that affect wellbeing, and the happiest people are those in close loving relationships (Diener \& Seligman, 2002). Good relationships correlate with mental health as well as physical health (Holt-Lunstad \& Smith, 2012). Understanding this connection and identifying ways to enable relationships to be at their most fulfilling has been a growing area of interest within PP, with studies showing that couples who increase their gratitude (Mikulincer, Shaver \& Slav, 2006), forgiveness (Fincham, 2015) or hope (Lavy \& Littman-Ovadia, 2011) find that it benefits their relationship, and their wellbeing improves as a result. This theory is appealing to those who find themselves in unsatisfactory relationships since it offers the promise that improvement is within their own power.

Domestic Abuse (DA) is defined by the UK government as: "any incident of controlling, coercive, threatening behavior, violence or abuse between those aged 16 or over who are, or have been intimate partners or family members regardless of gender or sexuality. This can encompass, but is not limited to the following types of abuse: psychological, physical, sexual, financial, emotional. Controlling behavior is a range of acts designed to make a person subordinate and/or dependent by isolating them from sources of support, exploring their resources and capacities for personal gain, depriving them of the means needed for independence, resistance and escape and 
regulating their everyday behavior. Coercive behavior is an act or pattern of acts of assaults, threats, humiliation and intimidation or other abuse that is used to harm, punish or frighten their victim." (Strickland \& Allan, 2017: p. 4)

DA affects 26.3\% of women in England and Wales (Strickland \& Allan, 2017) and a third of all women worldwide (Women UN, 2014). Women are more likely than men to experience DA, though it affects those from all genders, races, religious backgrounds and social classes (Strickland \& Allan, 2017). However, the extent of the problem remains largely unacknowledged by society, as it is a crime that takes place behind closed doors and is therefore easy to ignore. Often victims themselves will not recognize that they are experiencing abuse, and may downplay the seriousness of their situation (Walker, 2016).

Abuse often starts with psychological manipulation which can be subtle, but tends to escalate over time (Natarajan, 2017) both in severity and frequency, and in some cases results in murder: two women are killed by their intimate partner every week in the UK (ONS, 2016). The danger of death is higher still, since one in four victims of DA attempts suicide (Sansone, Chu, \& Wiederman, 2007). An abusive relationship is not consciously accepted by victims, however they may become accustomed to the escalating abuse, and therefore view their situation as normal. This can be explained by the Hedonic Adaptation Theory (Diener, Lucas \& Napa-Scollon, 2009) which is a mechanism that reduces the emotional impact of events that happen habitually. The frog in boiling water parable is also relevant here - if a live frog is dropped into a pan of boiling water, it would immediately jump out. However, if the frog is put into a pan of cool water which is gradually brought to the boil, the frog will stay in the water and be boiled to death.

Psychological abuse is difficult to identify as it does not leave marks in the way physical abuse does. Abusers often deny their actions, insinuating that the victim imagined the abuse. This is known as 'gaslighting' (Dorpat, 2006) and is particularly toxic as it makes victims doubt their own perceptions. Consequently, after unknowingly enduring DA, many victims suffer its symptoms without recognizing the cause. These symptoms include low mood, low energy, poor self-image, self-worth or confidence, and inability to think clearly (Dillon, Hussain, Loxton, \& Rahman, 2013; Devries, et al., 2013; Lagdon, Armour, \& Stringer, 2014). These symptoms can be easily misdiagnosed as mental illness, including anxiety (Dutton \& Painter, 1993), depression (Chang, Kahle \& Hirsch, 2015; Chang, Kahle, Yu \& Hirsch, 2014; O'Leary, 1999; Sackett \& Saunders, 1999) and posttraumatic stress disorder (Enns, Campbell \& Courtois, 1997.; Woods, 2000; Pimlott-Kubiak \& Cortina, 2003), and hence victims of DA are often prescribed anti-depressants as treatment. However, this does not solve the root problem and only leads to a more compliant victim.

DA was brought to the public attention by the feminist movement in the 1970's (Wendt, 2016). It centers on power imbalance causing some people to feel entitled to treat others who are less powerful than themselves disrespectfully and at times unlawfully. Gender differences in physical strength and economic advantage impact power dynamics. The fact that DA has been an ongoing problem throughout history suggests that gender inequality, the balance of power, and society's continued tolerance of this crime, has yet to be redressed.

\section{Positive Psychology Interventions and Domestic Abuse}


PP proposes that one's psychological wellbeing is largely within their own control (Lyubomirsky, 2013). A victim of DA who buys into this belief may choose to treat their symptoms by adopting Positive Psychology Interventions (PPIs) in an attempt to elevate their wellbeing (Fredrickson, 2001, 2009). Many PPIs can be used as mood boosting strategies even in clinical populations (Sin \& Lyubomirsky, 2009; Ruini, 2017; Layous, Chancellor \& Lyubomirsky, 2014). These interventions promise to benefit optimal wellbeing by retraining the mind into more positive habits. For example, gratitude, forgiveness, benefit finding and acts of kindness interventions (Emmons, 2007; McCullough \& van Oyen Witvliet, 2002; Affleck \& Tennen, 1996; Otake, Shimai, Tanaka-Matsumi, Otsui \& Fredrickson, 2006) can be particularly appealing for people who find themselves in difficult relationships. However, for DA victims, thinking themselves happy, is similar to taking antidepressants - it does not solve the problem of their toxic relationship, which can only be resolved by employing solution-focused strategies (Lazarus, 2003) to end the abuse. The abusers themselves can end the abuse by changing their attitude and behavior significantly, but sadly the likelihood of such behavior change is statistically very low (Day, Chung, O'Leary \& Carson, 2009). Failing to acknowledge and address the actual problem, and treating the symptoms, whether through medication or PPIs, only serves to pacify the victim who will then go on to suffer further cycles of escalating abuse.

McNulty (2010a) discovered that positive processes - such as positive expectations, attributions and behaviors, which are beneficial in healthy relationships, can be damaging in troubled relationships. For example, a spouse is disappointed when a disagreeable partner fails to live up to positive expectations, therefore it is more beneficial for wellbeing to expect disagreeable behavior and avoid disappointment. This follows the logic of defensive pessimism (Norem \& Chang, 2002) which involves mental preparation for challenging situations. McNulty and Fincham (2012) however demonstrated the importance of context for the success of PPIs: In their research on positive attitudes and wellbeing among newly married couples, they found that forgiveness promoted wellbeing in healthy relationships, but it was detrimental in unhealthy relationships. Strikingly, the study demonstrated that positivity increased the likelihood of the abuse reoccurring, as the abuser interprets the victim's forgiveness as tacit permission for continued abuse. These findings suggest that positivity may in effect aggravate DA.

Held (2002) voices concern that positivity can promote self-deception by creating a pleasant illusion of oneself and one's reality, and warns that this bubble can be burst by external events. Indeed, optimism has a dark side which Berlant (2011) describes as cruel because it can prompt people to endure violence and despair by providing the illusion of imminent improvement. Peterson (2000) argues that optimism can obscure reality therefore it is not always appropriate, and can backfire when the situation is not in one's control. The Optimistic Bias (Sharot, 2011) is a universally observed cognitive bias that prompts individuals to overestimate the likelihood of positive outcomes and underestimate the possibility of experiencing adverse events. This happens in spite of provision of information detailing risk statistics (Sharot, Korn \& Dolan, 2011). This bias is understandable as it would be unhelpful to live in constant fear.

Applying the principle of optimistic bias within the context of intimate relationships would mean favoring the belief that relationships are healthy and fulfilling, while ignoring the risk that they could be toxic or dangerous. Realism in place of unrealistic optimism, would enable a clearer 
assessment of a relationship's status as healthy or unhealthy, however this only seems possible at the cost of trust. Intimate relationships require a degree of trust, and suspicion does not foster closeness (Murray et al., 2013). Impulsive trust is a cognitive bias (Norris \& Zweigenhaft, 1999) which induces blind faith in a relationship's merit, as the alternative is not appealing to contemplate. This can explain why DA victims may display trust that their abusers have good intentions. The trust a victim has towards their abuser is further illustrated by the phenomenon of Stockholm Syndrome (Cantor \& Price, 2007) where a traumatized person develops a strong bond with their abuser. This happens when the abuser occasionally shows apparent compassion, e.g. a kidnapper giving their prisoner food. In contrast to the cruelty, any gesture of kindness is amplified and received with excessive gratitude. Stockholm Syndrome is interpreted as a coping mechanism (Speckhard et al., 2005) that enables a victim to survive their ordeal, although to an outsider this gratitude appears misplaced.

DA tends to occur in a repeating cycle (Walker, 2016): abuse - reconciliation - calm - tension building - abuse. In the periods of reconciliation and calm a victim often has positive feelings towards their abuser. The Stockholm Syndrome and the Cycle of Abuse help explain why DA victims often appear content with their situation because they are grateful for the intermittent good times. Gratitude and forgiveness are generally beneficial in close relationships (Lambert \& Fincham, 2011), but the caveat is that they are healthy relationships (McNulty \& Fincham, 2012). However, as it is difficult for a DA victim to discern the reality of their situation, they may attempt to improve their relationship by being more grateful, optimistic, empathetic and forgiving. Sadly, rather than facilitating positive change, this effort may fuel denial of their emotions, as well as denial of the DA and prolong their exposure to abuse.

\section{The Current Study}

This review aims to raise awareness of DA within the discipline of $\mathrm{PP}$, demonstrating the need for ethical consideration when advocating PPIs to the general public, as this will likely include individuals unwittingly suffering from DA. PP currently views DA as a 'clinical' concern which belongs in the domain of clinical psychology. However, due to its prevalence, DA is not merely a minority issue. Furthermore, as DA victims are often unconscious of their reality, they may be attracted to PPIs which promise to improve wellbeing and relationship satisfaction. Particularly alluring are PPIs employing gratitude, acceptance, optimism, hope, empathy and forgiveness which can cause inadvertent harm in the context of DA. Such PPIs could be considered avoidant coping in this context as they fail to acknowledge and address the problem itself. Furthermore, if symptoms of DA are mistaken for mental dysfunction for which the victim is treated, this not only fails to tackle the problem itself but it wrongly places the 'blame' on the victim.

Although DA is an important issue, there is little literature on the subject within PP. Therefore, this study aims to raise awareness of the specific problems faced by DA victims who may employ a variety of coping mechanisms which fall under the umbrella of positivity, and demonstrate that it is imperative that PPIs are not used without applying ethical considerations of the context in which they are applied. The research question posed is: can positivity be counterproductive when suffering DA? This question can be extrapolated to further circumstances 
of adversity, inequality or exploitation where positivity may also be disadvantageous. This question was explored by means of a narrative review, as the next section elucidates.

\section{METHOD}

\section{Narrative Reviews}

Over recent years, narrative reviews have become an increasingly popular tool for exploring and summarizing the literature on a given topic. In that respect, it is seen as having strengths that a more conventional systematic review lacks (and vice versa): for instance, whereas the latter by nature have more formalized and clearly-delineated search parameters, they may be less suited to tracking 'the development of a scientific principle or clinical concept,' with a risk that the 'narrative thread could be lost in the restrictive rules' mandated by such a review (Ferrari, 2015, p.230). In such instances, a narrative review may be more appropriate, as this affords more flexibility and freedom in appraising a given body of literature, while still maintaining the commitment to methodological comprehensiveness and analytical rigor that makes systematic reviews so valuable. Indeed, narrative reviews have already been used successfully in the context of analyzing DA, with Ali and Naylor (2013) exploring feminist, social, and ecological explanations for its causation. Similarly, in the present case, although a systematic review was initially considered as an approach, ultimately a narrative review was deemed more appropriate. This meant for example that although the search was conducted by using a pre-defined set of keywords, the researchers had latitude in how they assessed the nature of the paper (e.g., in terms of whether it did indeed focus on DA and positivity), and hence whether it was included in the analysis, and in what way it was interpreted and analyzed.

\section{Search Strategy}

The aim of this review was to find studies that indicate a correlation between 'positivity' and 'denial of DA'. For this purpose, a literature search was conducted using three electronic databases: PsychINFO, Social Care Online and Science Direct. These were selected because they span a variety of academic disciplines including Psychology and Social Work. The search took place in 2018 and was updated in April 2019.

\section{Data Items}

\section{Positivity}

In the context of this review 'positivity' was defined as the presence of an attitude or outlook which is generally considered to be beneficial in promoting wellbeing and improving interpersonal relationships: such as optimism, hope, acceptance, forgiveness, resilience, self-regulation, gratitude and empathy.

\section{Denial of DA}

The phenomenon of 'denial of $\mathrm{DA}^{\prime}$ is defined for the purpose of this review as failing to properly acknowledge or address a real risk of DA in terms of the absence of suitable problem-solving action. 'denial' represents the criticism of positivity for creating a false reality based on over-optimism or self-deception (Held, 2002; Jopling, 1996). The review judged studies as demonstrating this phenomenon if they showed the presence of an unrealistic risk assessment regarding DA and/or failing to take appropriate action to address DA in one's own personal life - particularly when this 
was despite provision of evidence to suggest a significant risk, or despite witnessing or withstanding behaviors considered to be unhealthy within the context of an intimate relationship. As DA follows the pattern of escalating over time, smaller acts of aggression are precursors to DA.

The phenomenon of 'Domestic Abuse' follows the Home Office definition (Strickland \& Allan, 2017: p. 4) which outlines in detail the context and behaviors which legally constitute DA.

\section{Keywords}

The keywords chosen for the literature search were: 'Domestic Abuse'; 'Domestic Violence'; 'Intimate Partner Violence'; 'Spousal Abuse'; 'Psychological Abuse'; and 'Emotional Abuse'.

The keywords representing 'Positivity' were selected because of their association with wellbeing as well as the belief that such attitudes improve interpersonal relationships. These were: 'Optimism'; 'Hope'; 'Acceptance'; 'Forgiveness'; 'Resilience'; 'Self-

Regulation'; 'Gratitude'; and 'Empathy'.

\section{Eligibility Criteria}

This review considered studies using a broad range of participants who were required to be above the age of 13 (participants above this age were judged to be sufficiently mature to consider the risk of DA). Participants could be of any gender, sexual orientation, ethnicity, race or social class. The study was particularly interested in participants who were DA victims or survivors. To clarify, 'victims' of DA refers to a person who is experiencing DA. Other participants of interest were couples, as this review recognized the potential for DA in relationships where aggressive behavior was observed. Other participants of interest were individuals contemplating their risk of experiencing DA.

\section{Study Selection and Data Collection}

The search systematically worked through each of the combination of keywords, in order to find quantitative studies which used a correlational research designs (reporting on correlations between variables or regression analyses). All other types of research designs were excluded from the review. Each of the eligible papers was read carefully and its data was extracted and entered into a table (see Table 1 next page) which included: details of the participants sampled in the study; the measures used to collect the data; and a summary of the findings. 
Table 1

The correlates of positivity and denial in DA in the literature

\begin{tabular}{|c|c|c|c|}
\hline PAPER & PARTICIPANTS & MEASURES & RESULTS \\
\hline $\begin{array}{l}\text { Chapin \& } \\
\text { Coleman } \\
(2009)\end{array}$ & $\begin{array}{l}\mathrm{N}=378 \text { hospital } \\
\text { staff attending DA } \\
\text { training USA. } \\
\text { Average age } 32, \\
85 \% \text { female, } 97 \% \\
\text { white. }\end{array}$ & $\begin{array}{l}\text { Optimistic Bias (Weinstein 1980) } \\
\text { Experience with DA (Carlson \& } \\
\text { Worden, 2005) } \\
\text { Attitude towards DA (Carlson \& } \\
\text { Worden, 2005) } \\
\text { Prior knowledge on DA } \\
\text { (developed for study) } \\
\text { Prevalence of DA in community } \\
\text { (participants' estimation) }\end{array}$ & $\begin{array}{l}\text { Staff perceived that they were less at } \\
\text { risk than others in their age group of } \\
\text { becoming victims of DA despite } \\
\text { training on the prevalence of DA. }\end{array}$ \\
\hline $\begin{array}{l}\text { Chapin \& } \\
\text { Coleman } \\
(2012)\end{array}$ & $\begin{array}{l}\mathrm{N}=601 \text { American } \\
\text { teenagers aged } 13- \\
16,54 \% \text { female, } \\
85 \% \text { white. }\end{array}$ & $\begin{array}{l}\text { Optimistic Bias (Weinstein 1980) } \\
\text { Self-esteem (Rosenberg, 1979) } \\
\text { Experience of DA (Carlson \& } \\
\text { Worden, 2005) } \\
\text { Prior knowledge on DA } \\
\text { (developed for study) } \\
\text { Media influence on others are on } \\
\text { oneself (developed for study) }\end{array}$ & $\begin{array}{l}\text { Teenagers demonstrated a optimistic } \\
\text { bias, believing they were less likely } \\
\text { than others to become victims of } \\
\text { violence by partner, despite some } \\
\text { reporting first-hand experience with } \\
\text { violence. }\end{array}$ \\
\hline $\begin{array}{l}\text { Chapin \& } \\
\text { Coleman } \\
(2014)\end{array}$ & $\begin{array}{l}\mathrm{N}=176 \text { American } \\
\text { teenagers aged } 13- \\
15,52 \% \text { female, } \\
95 \% \text { white. }\end{array}$ & $\begin{array}{l}\text { Optimistic Bias (Weinstein 1980) } \\
\text { Health Belief Model (developed } \\
\text { for study) } \\
\text { Perceived media reality measures } \\
\text { the perceived realism of TV shows } \\
\text { on DA (developed for study) }\end{array}$ & $\begin{array}{l}\text { Teenagers demonstrated a believe that } \\
\text { they are less likely than others to } \\
\text { become victims of family violence. } \\
\text { optimistic bias increased both as } \\
\text { perceived severity of violence } \\
\text { increased, and as perceived media } \\
\text { reality increased. }\end{array}$ \\
\hline $\begin{array}{l}\text { Chang et } \\
\text { al., (2014) }\end{array}$ & $\begin{array}{l}\mathrm{N}=101 \text { adults } \\
(28 \% \text { male) USA } \\
\text { Mean age of } 42 . \\
93 \% \text { of participants } \\
\text { were white. }\end{array}$ & $\begin{array}{l}\text { Domestic Abuse: HITS scale } \\
\text { (Sherin et al, 1998). } \\
\text { Forgiveness of Self and } \\
\text { Forgiveness of Others scales } \\
\text { (Mauger et al., 1992) } \\
\text { Suicide Behaviors } \\
\text { Questionnaire-Revised (SBQ-R) } \\
\text { (Osman et al., 2001) }\end{array}$ & $\begin{array}{l}33.3 \% \text { reported experiencing domestic } \\
\text { abuse at a fairly frequent rate, and } \\
32.4 \% \text { reported suicidal } \\
\text { thoughts or behaviors at a high } \\
\text { frequency. DA was associated with } \\
\text { suicide behavior. Forgiveness of self } \\
\text { and of others were significantly and } \\
\text { negatively associated with suicide } \\
\text { behavior. }\end{array}$ \\
\hline $\begin{array}{l}\text { Chang et } \\
\text { al., (2018) }\end{array}$ & $\begin{array}{l}\mathrm{N}=101 \text { adults ( } 29 \\
\text { males, } 72 \text { females) } \\
\text { from primary care } \\
\text { clinic in Southeast } \\
\text { United States }\end{array}$ & $\begin{array}{l}\text { HITS a four-item self-report } \\
\text { measure that assesses frequency } \\
\text { of partner abuse (developed for } \\
\text { study) } \\
\text { Hope Scale (Snyder et al., 1991) }\end{array}$ & $\begin{array}{l}\text { Hope is not always a protective factor } \\
\text { against suicidal behaviors for DA } \\
\text { victims. Specific analyses indicated } \\
\text { that having more hope may increase } \\
\text { risk for suicidal behaviors when an }\end{array}$ \\
\hline
\end{tabular}




\begin{tabular}{|c|c|c|c|}
\hline & & $\begin{array}{l}\text { Suicidal Behaviors } \\
\text { Questionnaire-Revised (Osman } \\
\text { et al., 2001) }\end{array}$ & $\begin{array}{l}\text { individual has experienced greater } \\
\text { amounts of DA. }\end{array}$ \\
\hline $\begin{array}{l}\text { Faramarzi } \\
\text { et al., } \\
(2005)\end{array}$ & $\begin{array}{l}\mathrm{N}=274 \text { Iranian } \\
\text { women, married, } \\
\text { aged } 20+, 17.9 \% \\
\text { pregnant. }\end{array}$ & $\begin{array}{l}\text { Abuse Assessment Screening } \\
\text { Form (developed for study) } \\
\text { Abuse Definition Form } \\
\text { (developed for study) } \\
\text { Abuse Attitude Form (developed } \\
\text { for study) }\end{array}$ & $\begin{array}{l}\text { Women with positive attitude to male } \\
\text { dominance accepted physical and } \\
\text { emotional abuse from their partner } \\
\text { more readily than those with a } \\
\text { negative attitude toward it. The } \\
\text { strongest predictor of physical abuse } \\
\text { was the women's attitude to male } \\
\text { dominance. }\end{array}$ \\
\hline $\begin{array}{l}\text { Fincham \& } \\
\text { Beach } \\
\text { (2002) - } \\
\text { Study } 1\end{array}$ & $\begin{array}{l}\mathrm{N}=88,44 \text { British } \\
\text { couples in the first } \\
\text { year of marriage. } \\
\text { Husbands average } \\
\text { age } 30 \text { and wives } \\
28 .\end{array}$ & $\begin{array}{l}\text { Specific Aggression Scale } \\
\text { (O'Leary \& Curly, 1986) } \\
\text { Marital Adjustment Test (Locke } \\
\text { \& Wallace, 1959) } \\
\text { Positive and negative } \\
\text { dimensions of forgiveness - } \\
\text { positive action tendency } \\
\text { (forgiving partner) and negative } \\
\text { tendency (retaliating) (developed } \\
\text { for study) }\end{array}$ & $\begin{array}{l}\text { Husbands reported greater } \\
\text { psychological aggression towards a } \\
\text { non-retaliating. Forgiveness has } \\
\text { neither purely a positive nor negative } \\
\text { impact in relationships, it can be } \\
\text { either. Psychological aggression was } \\
\text { found to decrease wellbeing. }\end{array}$ \\
\hline $\begin{array}{l}\text { Fincham \& } \\
\text { Beach } \\
\text { (2002) - } \\
\text { Study } 2\end{array}$ & $\begin{array}{l}\mathrm{N}=132,66 \text { British } \\
\text { couples. Husbands } \\
\text { average age } 32 \text { and } \\
\text { wives } 30 .\end{array}$ & $\begin{array}{l}\text { Specific Aggression Scale } \\
\text { (O'Leary \& Curly, 1986) } \\
\text { Marital Adjustment Test (Locke } \\
\text { \& Wallace, 1959) } \\
\text { Communication Patterns } \\
\text { Questionnaire (Futris et al., 2010) } \\
\text { Forgiveness (developed for } \\
\text { study) }\end{array}$ & $\begin{array}{l}\text { Actively forgiving was related to } \\
\text { constructive discussion of marital } \\
\text { problems. Not retaliating) predicted } \\
\text { reports of further psychological } \\
\text { aggression. }\end{array}$ \\
\hline $\begin{array}{l}\text { Gilbert \& } \\
\text { Gordon } \\
(2017)\end{array}$ & $\begin{array}{l}\mathrm{N}=121 \mathrm{USA} \\
\text { women in } 9 \mathrm{DA} \\
\text { shelters. Average } \\
\text { age } 34,82 \% \text { white. }\end{array}$ & $\begin{array}{l}\text { Conflict Tactics Scale (Straus, } \\
\text { 1979) } \\
\text { Silencing the Self Scale (Jack, } \\
\text { 1991) } \\
\text { Acts of Forgiveness Scale } \\
\text { (Drinnon, 2001) } \\
\text { Commitment Inventory (Stanley } \\
\text { \& Markman, 1992) }\end{array}$ & $\begin{array}{l}\text { Denial of injury was positively } \\
\text { correlated with commitment, as well } \\
\text { as with levels of forgiveness. The } \\
\text { higher a woman's commitment, the } \\
\text { more likely she would deny the } \\
\text { severity of her injury, which makes her } \\
\text { more likely to forgive her partner. }\end{array}$ \\
\hline $\begin{array}{l}\text { Gordon et } \\
\text { al. (2004) }\end{array}$ & $\begin{array}{l}\mathrm{N}=121 \text { women in } \\
\text { DA shelters. } \\
\text { Average age } 34 . \\
56 \% \text { married to/in } \\
\text { committed }\end{array}$ & $\begin{array}{l}\text { Commitment Inventory (Stanley } \\
\text { \& Markman, 1992) } \\
\text { Conflict Tactics Scale (Straus, } \\
\text { 1979) }\end{array}$ & $\begin{array}{l}\text { Women who are more forgiving of } \\
\text { their partner (less ruminative about } \\
\text { the abuse, less resentful / bitter) are } \\
\text { more likely to report intending to } \\
\text { return to them. }\end{array}$ \\
\hline
\end{tabular}


relationship

with/still seeing

perpetrator.
Acts of Forgiveness Scale

(Drinnon, 2001)

Domestic violence attributions

(developed for study)

The Intent to Return

Questionnaire (developed for study)

\begin{tabular}{|c|c|c|c|}
\hline $\begin{array}{l}\text { Katerndahl } \\
\text { et al. (2015) }\end{array}$ & $\begin{array}{l}\mathrm{N}=200 \text { women } \\
\text { with history of } \\
\text { physical DA. }\end{array}$ & $\begin{array}{l}\text { Religious Coping: COPE (Carver } \\
\text { et al., 1989) } \\
\text { Bioipsychosociospiritual } \\
\text { Inventory (Katerndahl \& } \\
\text { Oyiriaru, 2007) } \\
\text { Health Care Utilization Form } \\
\text { (Katerndahl \& Reulini, 1997) } \\
\text { Herth Hope Scale (1992) } \\
\text { Appraisal Dimension Scale } \\
\text { (Vitaliano, 1985) } \\
\text { Duke Social Support and Stress } \\
\text { Scale (Duke, 1986) }\end{array}$ & $\begin{array}{l}\text { Use of spiritual resources had variable } \\
\text { effect on DA victims, however } \\
\text { religious coping was associated with } \\
\text { refraining from leaving the } \\
\text { relationship. }\end{array}$ \\
\hline $\begin{array}{l}\text { Lahav et } \\
\text { al. (2019) }\end{array}$ & $\begin{array}{l}\mathrm{N}=520 \text { male } \\
\text { combat veterans } \\
\text { and } 171 \text { of their } \\
\text { female spouses, } \\
\text { average age } 51\end{array}$ & $\begin{array}{l}\text { 8-year longitudinal study } \\
\text { Conflict Tactics Scale (Straus, } \\
\text { 1979) } \\
\text { Family Forgiveness Scale } \\
\text { (Pollard et al., 1998) }\end{array}$ & $\begin{array}{l}\text { Forgiveness was a protective factor } \\
\text { among spouses experiencing low level } \\
\text { abuse, but its effect was non- } \\
\text { significant among those who suffered } \\
\text { higher levels of abuse. }\end{array}$ \\
\hline $\begin{array}{l}\text { Luchies et } \\
\text { al., (2010a, } \\
\text { 2010b) - } \\
\text { Study } 1\end{array}$ & $\begin{array}{l}\mathrm{N}=144,72 \text { USA } \\
\text { couples in first } 6 \\
\text { months of } \\
\text { marriage. }\end{array}$ & $\begin{array}{l}\text { 5-year longitudinal study } \\
\text { Transgression Narrative Test of } \\
\text { Forgivingness (Berry et al., 2001) } \\
\text { Agreeableness (Goldberg, 1999) } \\
\text { Rosenberg Self-Esteem Scale } \\
\text { (1965a) }\end{array}$ & $\begin{array}{l}\text { For individuals scoring high on } \\
\text { forgiveness whose spouse was low in } \\
\text { agreeableness, greater forgiveness } \\
\text { predicted their diminished self- } \\
\text { respect. }\end{array}$ \\
\hline $\begin{array}{l}\text { Luchies et } \\
\text { al. (2010a, } \\
\text { 2010b) - } \\
\text { Study } 2\end{array}$ & $\begin{array}{l}\mathrm{N}=49 \mathrm{USA} \\
\text { undergraduates. } \\
\text { Average age 20, } \\
55 \% \text { women, } 61 \% \\
\text { white. }\end{array}$ & $\begin{array}{l}\text { Forgiveness Manipulation } \\
\text { (Karremans et al., 2003) } \\
\text { Self-respect and self-concept } \\
\text { clarity (developed for study). }\end{array}$ & $\begin{array}{l}\text { Forgiving without receiving amends } \\
\text { caused significantly diminished self- } \\
\text { concept clarity. }\end{array}$ \\
\hline $\begin{array}{l}\text { Luchies et } \\
\text { al. (2010a, } \\
\text { 2010b) - } \\
\text { Study } 3\end{array}$ & $\begin{array}{l}\mathrm{N}=247 \text { USA } \\
\text { undergraduates. } \\
\text { Average age } 19 \text {, } \\
57 \% \text { women, } 61 \% \\
\text { white. }\end{array}$ & $\begin{array}{l}\text { Hypothetical betrayal (Boon \& } \\
\text { Sulsky, 1997) Self- } \\
\text { respect and self-concept clarity } \\
\text { (developed for study) }\end{array}$ & $\begin{array}{l}\text { Anticipated self-respect and self- } \\
\text { concept clarity following forgiveness } \\
\text { of a perpetrator depended on them } \\
\text { making amends. }\end{array}$ \\
\hline
\end{tabular}




\begin{tabular}{|c|c|c|c|}
\hline $\begin{array}{l}\text { Luchies et } \\
\text { al. (2010a, } \\
\text { 2010b) - } \\
\text { Study } 4\end{array}$ & $\begin{array}{l}\mathrm{N}=58 \mathrm{USA} \\
\text { undergraduates. } \\
\text { Average age } 18, \\
55 \% \text { women, } 76 \% \\
\text { white. Dating for } \\
\text { average } 13 \text { months. }\end{array}$ & $\begin{array}{l}\text { 6-month study, biweekly yes/no } \\
\text { questionnaire developed for study } \\
\text { measuring: } \\
\text { Self-respect and self-concept } \\
\text { clarity } \\
\text { Partner transgressions } \\
\text { Betrayal distress } \\
\text { Forgiveness } \\
\text { Amends }\end{array}$ & $\begin{array}{l}\text { Forgiving a partner who made weak } \\
\text { amends for a highly distressing } \\
\text { betrayal was associated with } \\
\text { diminished self-respect and self- } \\
\text { concept clarity. }\end{array}$ \\
\hline $\begin{array}{l}\text { Martin et } \\
\text { al. (2000) }\end{array}$ & $\begin{array}{l}\mathrm{N}=70, \text { women in } \\
\text { DA shelter. } \\
\text { Average age } 27, \\
77 \% \text { black. In } \\
\text { relationship with } \\
\text { batterer for average } \\
5 \text { years. }\end{array}$ & $\begin{array}{l}\text { Participants asked to assess } \\
\text { likelihood that most battered } \\
\text { women return to the batterer at } \\
\text { some point, as well as rating the } \\
\text { likelihood of themselves returning } \\
\text { to their batterer }\end{array}$ & $\begin{array}{l}\text { Nearly all the battered women } \\
\text { perceived the possibility they would } \\
\text { return to the batter as unlikely, but } \\
\text { perceived most battered women as } \\
\text { being at a greater risk }\end{array}$ \\
\hline $\begin{array}{l}\text { Mason \& } \\
\text { Pulvirenti } \\
(2013)\end{array}$ & $\begin{array}{l}\mathrm{N}=18 \\
\text { representatives } \\
\text { from refugee } \\
\text { support services }\end{array}$ & $\begin{array}{l}\text { Qualitative study, interviews on } \\
\text { the topic of resilience among } \\
\text { refugees experiencing DA }\end{array}$ & $\begin{array}{l}\text { Communities 'paper over' the issue of } \\
\text { DA in an act of collective resilience in } \\
\text { order to save face. This prevents } \\
\text { individuals from seeking support } \\
\text { outside their community. }\end{array}$ \\
\hline $\begin{array}{l}\text { McNulty } \\
(2008)\end{array}$ & $\begin{array}{l}\mathrm{N}=142,72 \\
\text { newlywed couples. } \\
\text { Husbands average } \\
\text { age } 30, \text { with } \\
\text { average } 14 \text { years of } \\
\text { education. } \\
\text { Wives average age } \\
24, \text { with average } 15 \\
\text { years of education. }\end{array}$ & $\begin{array}{l}\text { 2-year longitudinal study. } \\
\text { Quality Marriage Index (Norton, } \\
\text { 1983) } \\
\text { Transgression Narrative Test of } \\
\text { Forgivingness (Berry et al., 2001) } \\
\text { Conflict Tactics Survey (Straus, } \\
\text { 1979) }\end{array}$ & $\begin{array}{l}\text { Spouses with partners who rarely } \\
\text { behaved negatively tended to remain } \\
\text { more satisfied over time if they were } \\
\text { more forgiving, but spouses with } \\
\text { partners who frequently behaved } \\
\text { negatively experienced steeper } \\
\text { declines in satisfaction when they were } \\
\text { more forgiving. }\end{array}$ \\
\hline $\begin{array}{l}\text { McNulty } \\
(2010 b)\end{array}$ & $\begin{array}{l}\mathrm{N}=170,135 \\
\text { newlywed couples. } \\
\text { Husbands average } \\
\text { age } 26 \text {, with } 16 \\
\text { years of education. } \\
\text { Wives average age } \\
24 \text { with } 18 \text { years of } \\
\text { education. } 91 \% \\
\text { white. }\end{array}$ & $\begin{array}{l}\text { 7-day diary study. Measurements } \\
\text { devised for study: } \\
\text { Negative behavior self- } \\
\text { assessment. } \\
\text { Forgiveness. } \\
\text { Partner transgression severity. }\end{array}$ & $\begin{array}{l}\text { Forgiving a partner's negative } \\
\text { behavior once was associated with an } \\
\text { increased probability that the partner } \\
\text { would repeat the negative behavior. }\end{array}$ \\
\hline
\end{tabular}




\begin{tabular}{|c|c|c|c|}
\hline $\begin{array}{l}\text { McNulty } \\
(2011)\end{array}$ & $\begin{array}{l}\mathrm{N}=144,72 \\
\text { newlywed USA } \\
\text { couples. Husbands } \\
\text { average age } 25 \\
\text { with } 14 \text { years of } \\
\text { education, } 93 \% \\
\text { white. } \\
\text { Wives average age } \\
24 \text { with } 15 \text { years of } \\
\text { education, } 96 \% \\
\text { white. }\end{array}$ & $\begin{array}{l}\text { 4-year longitudinal study. } \\
\text { Transgression Narrative Test of } \\
\text { Forgivingness (Berry et al., 2001) } \\
\text { Conflict Tactics Scale (Straus, } \\
\text { 1979) } \\
\text { Quality Marriage Index (Norton, } \\
\text { 1983) }\end{array}$ & $\begin{array}{l}\text { Partners of forgiving spouses reported } \\
\text { perpetrating acts of physical and } \\
\text { psychological aggression against their } \\
\text { partners which remained stable during } \\
4 \text { years of marriage. Partners of less } \\
\text { forgiving spouses reported decrease in } \\
\text { acts of physical and psychological } \\
\text { aggression over } 4 \text { years. }\end{array}$ \\
\hline $\begin{array}{l}\text { McNulty \& } \\
\text { Russell } \\
\text { (2016) - } \\
\text { Study } 1\end{array}$ & $\begin{array}{l}\mathrm{N}=382 \text { USA } \\
\text { dating individuals. } \\
\text { Average age } 31, \\
52 \% \text { women, } \\
\text { current } \\
\text { relationship } \\
\text { average } 6 \text { years. }\end{array}$ & $\begin{array}{l}\text { Tendency to Forgive Scale } \\
\text { (Brown, 2003) } \\
\text { Agreeableness (Goldberg, 1999) } \\
\text { Quality Marriage Index (Norton, } \\
\text { 1983) } \\
\text { Participants wrote about a } \\
\text { behavior which 'upsets or even } \\
\text { hurts' their partner and provided } \\
\text { numerical estimate of times they } \\
\text { would 'engage in this behavior } \\
\text { over next month.' }\end{array}$ & $\begin{array}{l}\text { Partner forgiveness led to a reduction } \\
\text { of subsequent offending among more } \\
\text { agreeable people but increased } \\
\text { subsequent offending among less } \\
\text { agreeable people. Forgiving a less } \\
\text { agreeable partner was detrimental to } \\
\text { the relationship. }\end{array}$ \\
\hline $\begin{array}{l}\text { McNulty \& } \\
\text { Russell } \\
\text { (2016) - } \\
\text { Study } 2\end{array}$ & $\begin{array}{l}\mathrm{N}=88,44 \text { couples } \\
\text { dating for average } \\
12 \text { months. Men } \\
\text { average age } 19, \\
\text { women average } \\
\text { age } 18.91 \% \text { white. }\end{array}$ & $\begin{array}{l}\text { Agreeableness (Goldberg, 1999) } \\
\text { Forgiveness manipulation (Fazio } \\
\text { et al., 1995) in which the words } \\
\text { 'forgive' and 'grudge' appeared. }\end{array}$ & $\begin{array}{l}\text { Relatively disagreeable people increase } \\
\text { transgressions against a partner they } \\
\text { perceive as forgiving, but decrease } \\
\text { transgressions against a partner who } \\
\text { was less forgiving. }\end{array}$ \\
\hline $\begin{array}{l}\text { McNulty \& } \\
\text { Russell } \\
\text { (2016) - } \\
\text { Study } 3\end{array}$ & $\begin{array}{l}\mathrm{N}=270,135 \text { USA } \\
\text { newlywed couples. } \\
\text { Husbands average } \\
\text { age } 26 \text { with } \\
\text { average } 16 \text { years of } \\
\text { education. } \\
\text { Wives average age } \\
24 \text { with average } 16 \\
\text { years of education. } \\
92 \% \text { white. }\end{array}$ & $\begin{array}{l}\text { 4-year longitudinal study. } \\
\text { Agreeableness (Goldberg, 1999) } \\
\text { Transgression Narrative Test of } \\
\text { Forgivingness (Berry et al., 2001) } \\
\text { Conflict Tactics Scale (Straus, } \\
\text { 1979) Narcissistic } \\
\text { Personality Inventory (Raskin \& } \\
\text { Terry, 1988) } \\
\text { Compassionate Love Scale } \\
\text { (Sprecher \& Fehr, 2005) }\end{array}$ & $\begin{array}{l}\text { The success of a spouse's forgiveness } \\
\text { depends on their partner's levels of } \\
\text { agreeableness. Agreeable partners } \\
\text { corrected their negative behavior } \\
\text { when forgiven but disagreeable } \\
\text { partners did not. }\end{array}$ \\
\hline
\end{tabular}




\begin{tabular}{|c|c|c|c|}
\hline $\begin{array}{l}\text { McNulty \& } \\
\text { Russell } \\
\text { (2016) - } \\
\text { Study } 4\end{array}$ & $\begin{array}{l}\mathrm{N}=140,70 \\
\text { newlywed couples } \\
\text { Husbands average } \\
\text { age } 33 ; 55 \% \text { with } \\
\text { bachelor's degree, } \\
28 \% \text { with graduate } \\
\text { degree. } \\
\text { Wives average age } \\
31 ; 69 \% \text { bachelor's } \\
\text { degree, and } 29 \% \\
\text { graduate degree. } \\
74.4 \% \text { white. }\end{array}$ & $\begin{array}{l}\text { Agreeableness (Goldberg, 1999) } \\
\text { Transgressions (developed for } \\
\text { study) } \\
\text { Anger of partner (developed for } \\
\text { study) } \\
\text { Motivation to refrain from } \\
\text { transgressions (developed for } \\
\text { study) } \\
\text { Commitment (Rusbult et al., 1998) }\end{array}$ & $\begin{array}{l}\text { Relatively agreeable partners } \\
\text { perpetrated fewer transgressions } \\
\text { against more forgiving spouses, } \\
\text { whereas relatively disagreeable } \\
\text { partners perpetrated more } \\
\text { transgressions against more forgiving } \\
\text { spouses. }\end{array}$ \\
\hline $\begin{array}{l}\text { Meadows } \\
\text { et al. } \\
(2005)\end{array}$ & $\begin{array}{l}\mathrm{N}=200 \text { black USA } \\
\text { women aged 18-59 } \\
\text { who had } \\
\text { experienced } \\
\text { physical or } \\
\text { nonphysical DA. } 2 \\
\text { groups: (1) women } \\
\text { who presented to } \\
\text { the hospital } \\
\text { following nonfatal } \\
\text { suicide attempt } \\
\text { (attempters, } n= \\
\text { 100) } \\
\text { (2) women with no } \\
\text { history of suicidal } \\
\text { behavior (controls, } \\
n=100 \text { ) }\end{array}$ & $\begin{array}{l}\text { Suicide Attempt Status } \\
\text { (Meadows et al., 2005) } \\
\text { Herth Hope Index (1992) } \\
\text { Spiritual Well-Being Scale } \\
\text { (Paloutzian \& Ellison, 1982) } \\
\text { Self-Efficacy Scale for Battered } \\
\text { Women (Varvaro \& Palmer, 1993) } \\
\text { Preliminary Strategic Approach } \\
\text { to Coping Scale (Hobfoll et al., } \\
\text { 1994) } \\
\text { Social Support Behaviors Scale } \\
\text { (Vaux et al., 1987) } \\
\text { Effectiveness of Obtaining } \\
\text { Resources Scale (Sullivan et al., } \\
\text { 1992) }\end{array}$ & $\begin{array}{l}\text { Seven protective factors against } \\
\text { suicide attempts are positively related } \\
\text { to one another; hope, spiritual } \\
\text { wellbeing, self-efficacy, coping, social } \\
\text { support from family members, social } \\
\text { support from friends, effectiveness of } \\
\text { obtaining resources. DA victims with } \\
\text { high levels of these seven protective } \\
\text { factors were less likely to attempt } \\
\text { suicide. }\end{array}$ \\
\hline $\begin{array}{l}\text { Neff \& } \\
\text { Geers } \\
(2013)\end{array}$ & $\begin{array}{l}\mathrm{N}=122,61 \text { couples. } \\
\text { Husbands average } \\
\text { age } 26 \text { with } \\
\text { average } 16 \text { years of } \\
\text { education. } \\
\text { Wives average age } \\
24 \text { with } 16 \text { years of } \\
\text { education. } \\
64 \% \text { Christian, } 85 \% \\
\text { white. }\end{array}$ & $\begin{array}{l}\text { 1-year longitudinal study } \\
\text { Quality of Marriage Index } \\
\text { (Norton, 1983) } \\
\text { Marital Problems Inventory } \\
\text { (Geiss \& O'Leary, 1981) } \\
\text { Life Orientation Test (Scheier \& } \\
\text { Carver, 1985) } \\
\text { Rosenberg Self-Esteem Scale } \\
\text { (1965a) } \\
\text { Eysenck Personality } \\
\text { Questionnaire (1978) }\end{array}$ & $\begin{array}{l}\text { Dispositional optimism is more } \\
\text { adaptive for marital wellbeing than } \\
\text { relationship-focused optimism which } \\
\text { appeared to be detrimental to the } \\
\text { relationship. Spouses who deny or } \\
\text { resist relationship conflicts also miss } \\
\text { the opportunity for those conflicts to } \\
\text { bring about positive change. }\end{array}$ \\
\hline
\end{tabular}




\begin{tabular}{|c|c|c|c|}
\hline $\begin{array}{l}\text { Tsang \& } \\
\text { Stanford } \\
(2007)\end{array}$ & $\begin{array}{l}\mathrm{N}=207 \\
\text { Couples in which } \\
\text { male was convicted } \\
\text { of DA. } 132 \text { women } \\
\text { and } 75 \text { male } \\
\text { partners. } \\
\text { Women aged 18-71, } \\
61 \% \text { black. } \\
\text { Men aged 19-71, } \\
69 \% \text { black. } \\
\text { Relationship } \\
\text { duration ranged } \\
\text { from } 5 \text { months - } 33 \\
\text { years. }\end{array}$ & $\begin{array}{l}\text { Personality Assessment } \\
\text { Inventory (Morey, 1991) } \\
\text { Interpersonal Reactivity Index } \\
\text { (Davis \& Oathout, 1987) } \\
\text { Transgression-Related } \\
\text { Interpersonal Motivations } \\
\text { Inventory (McCullough et al., } \\
\text { 1998) } \\
\text { Intrinsic and Extrinsic Religious } \\
\text { Orientation Scales (Allport \& } \\
\text { Ross, 1967) } \\
\text { Quest Religious Orientation } \\
\text { Scale (Batson \& Schoenrade, 1991) }\end{array}$ & $\begin{array}{l}\text { Empathy and religiousness in women } \\
\text { was positively related to forgiveness. } \\
\text { The extent that the offender was seen } \\
\text { as accountable for violence was } \\
\text { mediated by the woman's state } \\
\text { empathy. }\end{array}$ \\
\hline $\begin{array}{l}\text { Zhang et } \\
\text { al. (2012) }\end{array}$ & $\begin{array}{l}\mathrm{N}=215 \text { pregnant } \\
\text { Chinese women }\end{array}$ & $\begin{array}{l}\text { Most measures were developed } \\
\text { for the study: } \\
\text { Abuse Assessment Screen } \\
\text { Attitude of acceptance toward } \\
\text { violence } \\
\text { History of witnessing violence } \\
\text { Concern over the newborn's } \\
\text { health/gender } \\
\text { Social support from family and } \\
\text { friends } \\
\text { Coping style } \\
\text { Eysenck Personality } \\
\text { Questionnaire (1994) } \\
\text { Rosenberg Self-Esteem Scale } \\
\text { (1965b) } \\
\text { Edinburgh Postpartum } \\
\text { Depression Scale (Cox et al., } \\
\text { 1987). }\end{array}$ & $\begin{array}{l}\text { Higher prevalence of postnatal } \\
\text { depression was found among women } \\
\text { who had been abused during } \\
\text { pregnancy than among those who had } \\
\text { not. An attitude of acceptance towards } \\
\text { violence was positively correlated with } \\
\text { postnatal depression. }\end{array}$ \\
\hline
\end{tabular}

\section{Risk of Bias}

As befitting a narrative review (rather than a systematic review), this review does not claim to be a complete and exhaustive review of the literature on this subject, since that is not within its remit. Rather it sought to find sufficient evidence in the literature to allow a rigorous examination of the relevant literature and to answer the research question with confidence.

The types of participants in the studies varied, which is a factor that entails a risk of bias, however a broad sample is advantageous if findings are consistent across a variety of participants. Although some of the measurement tools were used across multiple studies, there was a variety of tools used, and many studies included assessment tools that were created specifically for that study. 
Therefore, while it is not feasible to compare the studies like-for-like, the studies build a picture of the phenomenon by approaching it from different angles. A common trend can be identified despite the differences in methods. When documenting the findings in the table, care was taken to remain faithful to the researchers' view of their results without adding additional interpretations. This was to mitigate potential bias in only finding results which affirmatively answered the research question. The table provides a clear overview of the scope of this review and the aggregate of its findings.

\section{RESULTS}

The keyword search yielded titles of 22 relevant papers including a total of 29 studies. Details of these studies are outlined in table 1 above, which provides an overview of the patterns and tendencies found. The similarities in the findings across the studies build a coherent picture of the ways in which positivity functions in the context of DA.

\section{Optimism}

Optimistic bias is clearly shown to skew an individual's assessment of their own risk of DA, despite provision of information regarding the statistical risk of DA as Chapin and Coleman (2009, 2012, 2014) demonstrate in their studies. The hospital staff who were given training on DA and the statistical risk that it poses, failed to absorb the information, and directly after training reported believing they were less at risk of DA than the general population (Chapin \& Coleman, 2009). Likewise, teenage participants believed they were less at risk of experiencing DA than others, despite some of them reporting having had first-hand experience of violence (Chapin \& Coleman, 2012). Strikingly, when the risk of DA was described as more serious and violent, the perceived risk diminished further (Chapin \& Coleman, 2014). When testing optimistic bias with DA survivors in a DA shelter, the same trend was observed (Martin et al., 2000). Despite comprehending the risk of returning to their abusers, these survivors believed they were at lower risk of returning to their abusive partners than others. Optimism directed specifically towards relationships was shown to be unhelpful (Neff \& Geers, 2013) since it entails denial of problems and failure to employ effective problem solving. However, this study also found that a broader form of optimism, one that was based on the reality of a situation rather than wishful thinking, could benefit relationships as it would enable problems to be worked on and overcome.

\section{Forgiveness}

The evocative titles 'The Doormat Effect' (Luchies et al., 2010a; 2010b) and 'Forgive and Forget, or Forgive and Regret?' (McNulty \& Russell, 2016) strongly communicate the finding that forgiveness is not always beneficial within troubled marriages. The evidence suggests that the benefits of forgiveness in close relationships is context dependent - particularly on the agreeableness of the forgiven partner. In their studies, McNulty and Russell (2016) found that forgiving an agreeable partner benefitted the relationship and led to fewer transgressions over time. However, forgiving a less agreeable partner was detrimental to the relationship and led to continued abuse. McNulty (2010b) observed a similar finding where forgiveness has resulted in increased likelihood of the partner reoffending and increased aggression towards their spouses (McNulty, 2011), whereas 
partners who were not forgiven decreased their aggression towards their spouses. Lahav et al., (2019) demonstrate that forgiveness loses its protective factor the higher the level DA experienced. While forgiveness is generally seen as a positive outcome, it has limitations as noted in Fincham and Beach's (2002) studies. The authors observed that spouses displaying the 'negative aspect of forgiveness' (not holding their partner accountable) appeared to give tacit consent to partners to perpetrate further psychological aggression towards them. Their second study, however suggested that the 'positive aspect of forgiveness' (actively forgiving partners) led to the possibility of constructive conversations and problem solving within the relationship. However, when a spouse does not receive a positive response to their act of forgiveness, it takes a toll on their wellbeing. This was observed in McNulty's (2008) study which showed that among spouses whose partners frequently behaved negatively, forgiveness was associated with a decline in their wellbeing. A similar effect was measured by Luchies et al. (2010a; 2010b) who showed that if a spouse forgives a partner who is low in agreeableness, their self-respect diminishes over time - hence the 'Doormat

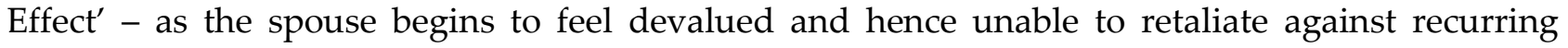
transgressions. However, the same study showed that forgiving an agreeable partner increased selfrespect over time.

\section{Acceptance}

Acceptance within the context of DA was shown to be generally detrimental and disempowering. Acceptance of a partner's violence increased the likelihood of a person staying in the relationship and tolerating this behavior (Faramarzi et al., 2005). Furthermore, acceptance of DA was correlated with higher instances of depression among DA victims (Zhang et al., 2012) showing that the act of acceptance in contrast to complaining or railing against one's unfortunate circumstances, does not improve wellbeing.

\section{Resilience}

Mason and Pulvirenti (2013) expose contradictions in how resilience functions and demonstrates its multidimensional nature; collective resilience vs. individual resilience. Often these dimensions can be at odds with each other. The study shows how the collective resilience of communities works to 'paper over' or downplay the problem of DA. This is an act of control over the individual to discourage them from seeking help outside their community which carries the perceived risk of shame for the entire community. The individual is coerced into relying on their own community as the only source of support and resilience which exacerbates abuse.

\section{Empathy}

Positivity was observed to be detrimental to DA victims and survivors, specifically regarding the risk of remaining with/returning to their abuser. Victims of physical forms of DA were shown to display greater denial of injury the more they were committed to their relationship (Gilbert \& Gordon, 2017). Women in DA shelters with a positive attitude (less rumination) were more likely to return to the offending partners (Gordon et al., 2004). Furthermore, religious coping - featuring forgiveness, empathy and hope - within the context of DA was associated with refraining from leaving the abusive relationship (Katerndahl et al., 2005). Empathy and religiousness in DA victims 
was related to forgiving the abuser (Tsang \& Stanford, 2007). Specifically, a victim's empathy mediated how they viewed their antagonist, eliciting greater sympathy towards them and the reasons behind their violent behavior. However, Meadows et al. (2005) demonstrated that positivity can benefit DA victims by protecting them from suicide. The study showed that a positive outlook (hope, spiritual wellbeing, and self-efficacy) was correlated with social support, as well as with obtaining resources needed to leave an abusive relationship. These findings suggest that a positive outlook within the context of DA - when coupled with social support - appears to guard against suicide. However, these findings are challenged by Chang et al. $(2014,2018)$ who found that forgiveness and hope were associated with suicide ideation and attempt.

\section{DISCUSSION}

Although positivity is beneficial in improving the quality of healthy relationships, there is ample evidence that within the context of troubled relationships a positive outlook can be unhelpful if not detrimental.

\section{Risk Assessment}

Positivity appears to promote denial of DA in terms of risk assessment (Chapin \& Coleman, 2009, 2012, 2014). This phenomenon was found in those experiencing abuse (Martin et al., 2000; Gordon et al., 2004; Gilbert \& Gordon, 2017; Faramarzi et al., 2005) which means that they were more likely to remain with their abuser. This is worrying because DA follows the pattern of escalating over time (Bland \& Ariel, 2015), and therefore the more time spent in an abusive situation, the greater the danger.

\section{Spiritual Bypassing}

The two studies which found a correlation between religiousness and refraining to leave an abusive relationship (Katerndahl et al., 2005; Tsang \& Stanford, 2007) demonstrate the principle of 'spiritual bypassing' (Cashwell et al., 2007). This mechanism attempts to heal problems at a spiritual level while ignoring practical considerations, therefore bypassing difficult emotions or tasks. A similar phenomenon is found in self-delusional positivity (Jopling, 1996) which cocoons people in an artificially sunny reality. This discourages people from changing unsatisfactory conditions as they put-up with them for longer than they would with a more realistic outlook (Berlant, 2011). Both spiritual bypassing and self-delusional positive thinking can be seen as avoidant-coping strategies because they entail seeking refuge from problems in belief systems. This may give the illusion of empowerment, when in fact escaping reality is disempowering (Jopling, 1996) since energy is siphoned from action-based problem-solving in the outside world, into the internal work of adjusting one's perceptions and suppressing undesirable emotions.

The crux of the problem with positivity is that it is often over-simplified and presented as an end in itself. For positivity to function optimally it needs to employ mind-set adjustment in tandem with practical problem-solving strategies. This is evidenced in the findings of Meadows et al. (2005) showing that a positive attitude together with the practical element of social support protects DA victims from suicide attempts. Neff and Geers (2013) also allude to this practical element of 
positivity, showing that although relationship-focused optimism was detrimental, a broader optimism which encompasses the wider reality of the situation does appear to be advantageous for working through marital problems. In the case of DA this could involve the realistic optimistic view that bringing an unhealthy relationship to an end would increase wellbeing. Fincham and Beach (2002) also show that a positive attitude needs to be coupled with action as their 'positive dimension of forgiveness' involved actively working on marital problems, in contrast to the 'negative dimension' which was passive.

\section{The Doormat Effect}

The review found a significant number of studies that show that forgiving a disagreeable partner increases the likelihood that they will transgress again (McNulty 2010b, 2011; McNulty \& Russell, 2016; Fincham \& Beach, 2002). This is particularly poignant to DA as incidents reoccur in a Cycle of Abuse (Walker, 2016) which keeps repeating until the cycle is broken. The effect of acting positively towards a partner but failing to get a positive response was shown to be detrimental to wellbeing (McNulty, 2008; Luchies et al., 2010a, 2010b; Zhang et al., 2012; Chang et al., 2015). Particularly compelling are the findings of Luchies et al. (2010a, 2010b) which show that forgiving a partner who continues to transgress leads to declining self-respect and loss of self-concept. This is consistent with symptoms of DA victims who suffer from loss of self as a result of being unseen and emotionally neglected (Kauffman, 2011) which is psychological damage that requires extensive therapy to correct. These findings (Luchies et al., 2010a; 2010b) demonstrate how unreciprocated positivity can contribute to loss of self-concept.

\section{DA Awareness}

DA is a social problem, and tacit group tolerance of DA hinders victims from finding the requisite support (Mason \& Pulvirenti, 2013) so addressing this is a vital step in tackling the problem. An informed perspective seeks to raise consciousness of DA as a societal issue (Wendt, 2016). This lessens shame and self-blame making it easier for victims to confront the problem and seek help. Continued efforts to raise awareness of DA, and of signs distinguishing healthy from unhealthy relationships is vital, but may take generations for society to assimilate. In the current social climate, many will inevitably continue to experience DA. Once abuse escalates to a point that it can no longer be excused, victims steeped in self-delusional positivity are liable find their 'bubble' of protective self-deception burst (Held, 2002) which although devastating, heralds the start of recovery. An event that shatters personal assumptions invites them to be reviewed, providing the opportunity to form a richer set of beliefs. This phenomenon is known as Post Traumatic Growth (Calhoun \& Tedeschi, 2010) where trauma invites the possibility of positive change and improved wellbeing. Providing that they are not re-victimized, DA survivors have been shown to display Post Traumatic Growth following a year of recovery (Valdez \& Lilly, 2015). The irony is that DA provides the circumstances for self-development by awakening victims from a naïve positivity into a more enriching, authentic positivity.

\section{Limitations of Study}


As this is a narrative review it did not include a formal analysis of quality as per the QATQS process used in systematic reviews. However, this review is important as it adds to the body of research exploring the importance of context for the success of PPIs, and highlights some of the challenges in distinguishing appropriate from inappropriate contexts. The review was limited to certain types of positivity as the keywords curtailed the number of papers discovered. In particular the search omitted investigation into the potential for trust to have negative consequences in close relationships, and to question whether it is possible for intimate relationships to exist without trust. This review also found no studies measuring the correlation between gratitude and denial of DA, but this is suggested in literature linking Stockholm Syndrome with DA (Cantor \& Price, 2007) as it assumes misplaced gratitude towards an abuser. Nor did the search find any research into the pitfalls of self-regulation within the context of abusive relationships. This indicates specific gaps in the research for future studies to explore.

\section{Second Wave PP}

The review affirmatively answers the research question and shows that the pressure to be positive can harm victims of DA. Positivity must not be confused with 'putting up with' unhealthy situations. Sometimes it is rational, justified and adaptive to complain or retaliate (Held, 2001) especially given that suppressing emotions can result in illness by compromising the body's immune system (Pennebaker, 1997). Second Wave PP (Lomas \& Ivtzan, 2015) argues that although PP is primarily concerned with positive outcomes, it is possible for positive-valenced emotions and processes to hinder wellbeing (as in the case of the present study), and conversely for negatively-valenced emotions and processes - such as boredom (Lomas, 2017), sadness (Lomas, 2018), or anger (Lomas, 2019 ) - to promote wellbeing. This emerging area of research within PP is a welcome addition, since it allows the entire spectrum of human emotion - including anger and sadness - to be accepted rather than ignored, therefore addressing the critique of PP as promoting the tyranny of positivity (Held, 2002).

\section{CONCLUSION}

The pressure to be positive can cause damage in particular contexts as it may discourage people from facing the reality of their situation. This results in denial both of the reality of difficult circumstances as well as the accompanying emotions, therefore forced-positivity can be an avoidant coping mechanism.

PP needs to take into account the dangers of applying PPIs within unsuitable contexts, assessing potential risks and understanding the restrictions on what positivity can remedy. There is a danger that problems may be minimized by categorizing them as merely requiring a change of perception. The positivity movement has gathered momentum in this direction, with many buying into the idea of the power of positivity. Self-help books within the PP arena can often allude to the promise of achieving a charmed life by changing one's mental attitude (even if the research literature on which these books are based is more nuanced). The appeal of such an undemanding solution is similar to the promise of financial security by purchasing a scratch-card, and no one is more tempted by this seductive offer than the vulnerable. In light of this exploitation of vulnerable people, even if unintentional, PP needs to use its position as expert adviser in order to influence the maturation of 
the positivity movement. PP should attempt to temper the current social pressure to be positive by imbuing it with reality and a greater sense of responsibility to its end users. This can be achieved by carrying out further research into the downsides of PPIs when applied in the context of harmful situations. Greater care should be taken by PP scholars and practitioners when extoling the virtues of positivity inducing exercises, acknowledging their limitations and emphasizing the important contributing factor of context in place of prescribing a one-size-fits-all approach to improving wellbeing.

\section{Authors}

Ellen Sinclair

University of East London

Rona Hart

r.hart@uel.ac.uk

University of East London

Tim Lomas

University of East London

\section{Publishing Timeline}

Received 9 July 2018

Accepted 16 November 2019

Published 1 February 2020

\section{References}

Affleck, G., \& Tennen, H. (1996). Construing benefits from adversity: Adaptotional significance and dispositional underpinnings. Journal of personality, 64(4), 899-922.

Ali, P. A., \& Naylor, P. B. (2013). Intimate partner violence: A narrative review of the feminist, social and ecological explanations for its causation. Aggression and Violent Behavior, 18(6), 611-619. doi: 10.1016/j.avb.2013.07.009

Allport, G. W., \& Ross, J. M. (1967). Personal religious orientation and prejudice. Journal of Personality and Social Psychology, 5, 432-443.

Batson, C. D., \& Schoenrade, P. A. (1991). Measuring religion as quest: (2) Reliability concerns. Journal for the Scientific Study of Religion, 30, 430-447.

Berlant, L. (2011). Cruel optimism. Durham, NC and London: Duke University Press

Berry, J. W., Worthington, E. L., Parrott, L., O'Connor, L. E., \& Wade, N. G. (2001). Dispositional forgivingness: Development and construct validity of the Transgression Narrative Test of Forgivingness (TNTF). Personality and Social Psychology Bulletin, 27, 1277-1290.

Bland, M., \& Ariel, B. (2015). Targeting escalation in reported domestic abuse: Evidence from 36,000 callouts. International criminal justice review, 25(1), 30-53. 
Bohart, A. C., \& Greening, T. (2001). Humanistic psychology and positive psychology. American Psychologist, 56(1), 81-82.

Boon, S. D., \& Sulsky, L. M. (1997). Attributions of blame and forgiveness in romantic relationships: A policy-capturing study. Journal of Social Behavior and Personality, 12, 19-44.

Brown, R. P. (2003). Measuring individual differences in the tendency to forgive: Construct validity and links with depression. Personality and Social Psychology Bulletin, 29, 759-771.

Calhoun, L. G., Cann, A., \& Tedeschi, R. G. (2010). The posttraumatic growth model: Sociocultural considerations. In T. Weiss, R. Berger, T. Weiss, R. Berger (Eds.), Posttraumatic growth and culturally competent practice: Lessons learned from around the globe (pp. 1-14). Hoboken, NJ, US: John Wiley \& Sons Inc.

Cantor, C., \& Price, J. (2007). Traumatic entrapment, appeasement and complex post-traumatic stress disorder: Evolutionary perspectives of hostage reactions, domestic abuse and the Stockholm syndrome. Australian and New Zealand Journal of Psychiatry, 41(5), 377-384.

Carlson, B. \& Worden, A. (2005). Attitudes and beliefs about domestic violence: Results of a public opinion survey. Journal of Interpersonal Violence, 20(10), 1197-1218.

Carver, C. S., Scheier, M. F., \& Weintraub, J. K. (1989). Assessing coping strategies: a theoretically based approach. Journal of personality and social psychology, 56(2), 267.

Cashwell, C. S., Bentley, P. B., \& Yarborough, J. P. (2007). The Only Way Out is Through: The Peril of Spiritual Bypass. Counseling and Values, 51(2), 139-148.

Chang, E. C., Yu, E. A., Kahle, E. R., Du, Y., Chang, O. D., Jilani, Z., \& Hirsch, J. K. (2018). The relationship between domestic partner violence and suicidal behaviors in an adult community sample: examining hope agency and pathways as protective factors. Violence against women, 24(12), 1399-1412

Chang, E. C., Kahle, E. R., \& Hirsch, J. K. (2015). Understanding how domestic abuse is associated with greater depressive symptoms in a community sample of female primary care patients: Does loss of belongingness matter?. Violence against women, 21(6), 700-711.

Chang, E. C., Kahle, E. R., Yu, E. A., \& Hirsch, J. K. (2014). Understanding the relationship between domestic abuse and suicide behavior in adults receiving primary care: Does forgiveness matter?. Social work, 59(4), 315-320.

Chapin, J., \& Coleman, G. (2014). Adolescents' perceptions of family violence risks. Journal of Family Violence, 29(7), 757-761.

Chapin, J. R., \& Coleman, G. (2012). Optimistic bias about dating/relationship violence among teens. Journal of Youth Studies, 15(5), 645-655.

Chapin, J., \& Coleman, G. (2009). Optimistic bias: What you think, what you know or who you know?. North American Journal of Psychology, 11(1), 121-132.

Coyne, J.C., and Tennen, H. (2010). Positive psychology in cancer care: Bad science, exaggerated claims, and unproven medicine. Annals of Behavioral Medicine, (39), 16-26.

Coyne, J.C., Tennen, H., and Ranchor, A.V. (2010). Positive psychology in cancer care: A story line resistant to evidence. Annals of Behavioral Medicine, (39), 35-42.

Cox, J.L., Holden. J.M., Sagovsky, R. (1987) Detection of postnatal depression. Development of the 10-item Edinburgh Postnatal Depression Scale. Br J Psychiatry, 150: 782-786. 
Davis, M. H., \& Oathout, H. A. (1987). Maintenance of satisfaction in romantic relationships: Empathy and relational competence. Journal of Personality and Social Psychology, 53, 397-410.

Day, A., Chung, D., O'Leary, P., \& Carson, E. (2009). Programs for men who ogetrate domestic violence: An examination of the issues underlying the effectiveness of intervention programs. Journal of Family Violence, 24(3), 203-212.

Devries, K.M., Mak, J.Y., Bacchus, L.J., Child, J.C., Falder, G., Petzold, M., Astbury, J., \& Watts, C.H., (2013). Intimate partner violence and incident depressive symptoms and suicide attempts: a systematic review of longitudinal studies. PLoS medicine, 10(5), p.e1001439.

Diener, E., \& Seligman, M. P. (2002). Very happy people. Psychological Science, 13(1), 81-84.

Diener, E., Lucas, R.E. \& Napa-Scollon, C., (2009). Beyond the hedonic treadmill: revisiting the adaptation theory of wellbeing. In E. Diener (Ed): The Science of Wellbeing (103-118), London: Springer.

Dillon, G., Hussain, R., Loxton, D., \& Rahman, S. (2013). Mental and physical health and intimate partner violence against women: A review of the literature. International journal of family medicine, Article ID 313909.

Dorpat, T. (2006). Covert methods of interpersonal control. In R. Raubolt (Ed.), Power games: Influence, persuasion, and indoctrination in psychotherapy training (pp. 93-118). New York, NY, US: Other Press.

Drinnon, J. E. R. (2001). Assessing forgiveness: Development and validation of the Act of Forgiveness Scale. Dissertation Abstracts International, 61 (6185), 11-B.

Duke (1986). Duke Social Support and Stress Scale. Durham: Duke University Press.

Dutton, D. G., \& Painter, S. (1993). The battered woman syndrome: Effects of severity and intermittency of abuse. American Journal of Orthopsychiatry,63, 614-621

Emmons, R. A. (2007). Thanks!: How the new science of gratitude can make you happier. Houghton Mifflin Harcourt.

Enns, C. Z., Campbell, J., \& Courtois, C. A. (1997). Recommendations for working with domestic violence survivors, with special attention to memory issues and post-traumatic processes. Psychotherapy, 34, 459-477.

Eysenck, H. J., \& Eysenck, S. B. G. (1994). Manual of the Eysenck Personality Questionnaire:(EPQ-R Adult). EdITS/Educational and Industrial Testing Service.

Faramarzi, M., Esmailzadeh, S. \& Mosavi, S. (2005). A comparison of abused and non-abused women's definitions of domestic violence and attitudes to acceptance of male dominance. European Journal of Obstetrics \& Gynecology and Reproductive Biology, 122, 225-231.

Fazio, R. H., Jackson, J. R., Dunton, B. C., \& Williams, C. J. (1995). Variability in automatic activation as an unobtrusive measure of racial attitudes: A bona fide pipeline? Journal of Personality and Social Psychology, 69, 1013-1027.

Ferrari, R. (2015). Writing narrative style literature reviews. Medical Writing, 24(4), 230-235. doi: 10.1179/2047480615Z.000000000329

Fincham, F. D., \& Beach, S. R. H. (2002). Forgiveness in marriage: Implications for psychological aggression and constructive communication. Personal Relationships, 9(3), 239-251.

Fincham, F. D. (2015). Forgiveness, family relationships and health. In L. L. Toussaint, E. J. Worthington, D. R. Williams, L. L. Toussaint, E. J. Worthington, D. R. Williams (Eds.), 
Forgiveness and health: Scientific evidence and theories relating forgiveness to better health (pp. 255270). New York, NY, US: Springer Science + Business Media.

Fredrickson, B. L. (2001). The role of positive emotions in positive psychology: The broaden-andbuild theory of positive emotions. American Psychologist, 56(3), 218-226.

Fredrickson, B. L. (2009). Positivity: Groundbreaking research reveals how to embrace the hidden strength of positive emotions, overcome negativity, and thrive. USA: Crown Publisher.

Futris, T. G., Campbell, K., Nielsen, R. B., \& Burwell, S. R. (2010). The Communication Patterns Questionnaire - Short Form: A review and assessment. The Family Journal, 18(3), 275-287.

Geiss, S. K., \& O'Leary, K. D. (1981). Therapist ratings of frequency and severity of marital problems. Journal of Marital and Family Therapy, 7, 515-520.

Gilbert, S. E., \& Gordon, K. C. (2017). Predicting forgiveness in women experiencing intimate partner violence. Violence Against Women, 23(4), 452-468.

Goldberg, L. R. (1999). A broad-bandwidth, public-domain, personality inventory measuring the lower-level facets of several five-factor models. In I. Mervielde, I. Deary, F. De Fruyt, \& F. Ostendorf (Eds.), Personality psychology in Europe (Vol. 7, pp. 7-28). Tilburg, The Netherlands: Tilburg University Press.

Gordon, K. C., Burton, S., \& Porter, L. (2004). Predicting the Intentions of Women in Domestic Violence Shelters to Return to Partners: Does Forgiveness Play a Role?. Journal of Family Psychology, 18(2), 331-338.

Held, B. S. (2002). The tyranny of the positive attitude in America: Observation and speculation. Journal of Clinical Psychology, 58(9), 965-992.

Held, B. S. (2001). Stop smiling, start kvetching; A 5-step guide to creative complaining. New York: St. Martin's Griffin.

Herth, K. (1992). Abbreviated instrument to measure hope: Development and psychometric evaluation. Journal of Advanced Nursing, 17, 1251-1259.

Hobfoll, S. E., Dunahoo, C. L., Ben-Porath, Y., \& Monnier, J. (1994). Gender and coping: The dual axis model of coping. American Journal of Community Psychology, 22, 49- 82.

Holt-Lunstad, J., \& Smith, T. B. (2012). Social relationships and mortality. Social And Personality Psychology Compass, 6(1), 41-53.

Jack, D. C. (1991). Silencing the self. New York: Harper Perennial.s[EP]

Jopling, D. A. (1996). 'Take away the life-lie ...': Positive illusions and creative self-deception. Philosophical Psychology, 9(4), 525-544.

Karremans, J. C., Van Lange, P. A. M., Ouwerkerk, J. W., \& Kluwer, E. S. (2003). When forgiving enhances psychological well-being: The roles of interpersonal commitment. Journal of Personality and Social Psychology, 84, 1011-1026.

Katerndahl, D., Burge, S., Ferrer, R., Becho, J., \& Wood, R. (2015). Effects of religious and spiritual variables on outcomes in violent relationships. International Journal of Psychiatry in Medicine, 49(4), 249-263.

Katerndahl, D., \& Oyiriaru, D. (2007). Assessing the Biopsychosociospiritual Model in primary care: Development of the Biopsychosociospiritual Inventory (BioPSSI). International Journal of Psychiatry in Medicine, 37(4), 393-414. 
Katerndahl, D. A., \& Realini, J. P. (1997). Comorbid psychiatric disorders in subjects with panic attacks. Journal of Nervous and Mental Disease, 185(11), 669-674.

Kauffman, J. (2011). The trauma of neglect: Loss of self. In D. L. Harris (Ed.) Counting our losses: Reflecting on change, loss, and transition in everyday life (pp.143-150). New York, NY, US: Routledge/Taylor \& Francis Group.

Lambert, N. M., \& Fincham, F. D. (2011). Expressing gratitude to a partner leads to more relationship maintenance behavior. Emotion, 11(1), 52-60.

Lagdon, S., Armour, C., \& Stringer, M. (2014). Adult experience of mental health outcomes as a result of intimate partner violence victimisation: a systematic review. European journal of psychotraumatology, 5(1), 24794.

Lahav, Y., Renshaw, K. D., \& Solomon, Z. (2019). Domestic abuse and forgiveness among military spouses. Journal of Aggression, Maltreatment $\mathcal{E}$ Trauma, 28(2), 243-260.

Lavy, S., \& Littman-Ovadia, H. (2011). All you need is love? Strengths mediate the negative associations between attachment orientations and life satisfaction. Personality and Individual Differences, 50(7), 1050-1055.

Lazarus, R. S. (2003). Does the Positive Psychology Movement Have Legs? Psychological Inquiry, 14(2), 93-109.

Layous, K., Chancellor, J., \& Lyubomirsky, S. (2014). Positive activities as protective factors against mental health conditions. Journal of Abnormal Psychology, 123(1), 3-12.

Locke, H. J., \& Wallace, K. M. (1959). Short marital adjustment prediction tests: Their reliability and validity. Marriage and Family Living, 21, 251-255.

Lomas, T. (2017). A meditation on boredom: Re-appraising its value through introspective phenomenology. Qualitative Research in Psychology, 14(1), 1-22. doi: 10.1080/14780887.2016.1205695

Lomas, T. (2018). The quiet virtues of sadness: A selective theoretical and interpretative appreciation of its potential contribution to wellbeing. New Ideas in Psychology, 49, 18-26. doi: 10.1016/j.newideapsych.2018.01.002

Lomas, T. (2019). Anger as a moral emotion: A 'bird's eye' systematic review. Counselling Psychology Quarterly. doi: 10.1080/09515070.2019.1589421

Lomas, T., \& Ivtzan, I. (2015). Second wave positive psychology: Exploring the positive-negative dialectics of wellbeing. Journal of Happiness Studies, 17, 1753-1768.

Luchies, L. B., Finkel, E. J., McNulty, J. K., \& Kumashiro, M. (2010a). The doormat effect: When forgiving erodes self-respect and self-concept clarity. Journal of Personality and Social Psychology, 98(5), 734-749.

Luchies, L. B., Finkel, E. J., McNulty, J. K., \& Kumashiro, M. (2010b). 'The doormat effect: When forgiving erodes self-respect and self-concept clarity': Correction to Luchies, Finkel, McNulty, and Kumashiro (2010). Journal of Personality and Social Psychology, 99(1), 119.

Lyubomirsky, S. (2001). Why are some people happier than others? The role of cognitive and motivational processes in well-being. American Psychologist, 56(3), 239-249.

Lyubomirsky, S. (2013). The myths of happiness. NY, NY: Penguin Press. 
Martin, A. J., Berenson, K. R., Griffing, S., Sage, R. E., Madry, L., Bingham, L. E., \& Primm, B. J. (2000). The process of leaving an abusive relationship: The role of risk assessments and decisioncertainty. Journal of Family Violence, 15(2), 109-122.

Mason, G., \& Pulvirenti, M. (2013). Former refugees and community resilience: 'Papering over' domestic violence. British Journal of Criminology, 53(3), 401-418.

Mauger, P. A., Perry, J. E., Freeman, T., Grove, D. C., McBride, A. G., \& McKinney, K. E. (1992). The measurement of forgiveness: Preliminary research. Journal of Psychology and Christianity, 11, 170-180.

McCullough, M. E., Rachal, K. C., Sandage, S. J., Worthington, E. L., Jr., Brown, S. W., \& Hight, T. L. (1998). Interpersonal forgiving in close relationships II: Theoretical elaboration and measurement. Journal of Personality and Social Psychology, 75, 1586-1603.

McCullough, M. E., \& Witvliet, C. V. (2002). The psychology of forgiveness. Handbook of positive psychology, 2, 446-455.

McNulty, J. K. (2008). Forgiveness in marriage: Putting the benefits into context. Journal of Family Psychology, 22(1), 171-175.

McNulty, J. K. (2010a). When positive processes hurt relationships. Current Directions in Psychological Science, 19(3), 167-171.

McNulty, J. K. (2010b). Forgiveness increases the likelihood of subsequent partner transgressions in marriage. Journal of Family Psychology, 24(6), 787-790.

McNulty, J. K. (2011). The dark side of forgiveness: The tendency to forgive predicts continued psychological and physical aggression in marriage. Personality and Social Psychology Bulletin, 37(6), 770-783.

McNulty, J. K. \& Fincham, F. D. (2012). Beyond positive psychology? Toward a contextual view of psychological processes and well-being. American Psychologist, 67(2). 101-110.

McNulty, J. K., \& Russell, V. M. (2016). Forgive and forget, or forgive and regret? Whether forgiveness leads to less or more offending depends on offender agreeableness. Personality and Social Psychology Bulletin, 42(5), 616-631.

Meadows, L. A., Kaslow, N. J., Thompson, M. P., \& Jurkovic, G. J. (2005). Protective Factors Against Suicide Attempt Risk Among African American Women Experiencing Intimate Partner Violence. American Journal of Community Psychology, 36(1-2), 109-121.

Mikulincer, M., Shaver, P. R., \& Slav, K. (2006). Attachment, mental representations of others, and interpersonal gratitude and forgiveness within romantic relationships. In M. Mikulincer \& G. S. Goodman (Eds.) Dynamics of romantic love (pp. 190-215). New York: Guilford Press.

Morey, L. C. (1991). Personality Assessment Inventory: Professional manual. Odessa, FL: Psychological Assessment Resources.

Murray, S. L., Gomillion, S., Holmes, J. G., Harris, B., \& Lamarche, V. (2013). The dynamics of relationship promotion: Controlling the automatic inclination to trust. Journal of Personality and Social Psychology, 104(2), 305-334.

Natarajan, M. (Ed.). (2017). Domestic violence: The five big questions. London: Routledge.

Neff, L. A., \& Geers, A. L. (2013). Optimistic expectations in early marriage: A resource or vulnerability for adaptive relationship functioning?. Journal of Personality and Social Psychology, 105(1), 38-60. 
Norem, J. K., \& Chang, E. C. (2002). The positive psychology of negative thinking. Journal of clinical psychology, 58(9), 993-1001.

Norris, S. L., \& Zweigenhaft, R. L. (1999). Self-monitoring, trust, and commitment in romantic relationships. The Journal of Social Psychology, 139(2), 215-220.

Norton, R. (1983). Measuring marital quality: A critical look at the dependent variable. Journal of Marriage and the Family, 45, 141-151.

Office for National Statistics (2016). Homicide, London: ONS.

O'Leary, K. D., \& Curly, A. D. (1986). Assertion and family violence: Correlates of spouse abuse. Journal of Marital and Family Therapy, 12, 281-289.

O'Leary, D. K. (1999). Psychological abuse: A variable deserving critical attention in domestic violence. Violence and Victims, 14(1), 3-23.

Osman, A., Bagge, C. L., Gutierrez, P. M., Konick, L. C., Kooper, B. A., \& Barrios, F. X. (2001). The Suicidal Behaviors Questionnaire-Revised (SBQ-R): Validation with clinical and nonclinical samples. Assessment, 5, 443-454.

Otake, K., Shimai, S., Tanaka-Matsumi, J., Otsui, K., \& Fredrickson, B. L. (2006). Happy people become happier through kindness: A counting kindnesses intervention. Journal of happiness studies, 7(3), 361-375.

Paloutzian, R. F., \& Ellison, C. W. (1982). Loneliness, spiritual well-being, and the quality of life. In L. A. Peplau \& D. Perlman (Eds.), Loneliness: A sourcebook of current theory, research, and therapy (pp. 224-237). New York: Wiley.

Pennebaker, J. W. (1997). Opening up: The healing power of expressing emotions., Rev. ed. New York: Guilford Press.

Peterson, C. (2000). The future of optimism. American Psychologist, 55(1), 44-55.

Pimlott-Kubiak, S., \& Cortina, L. M. (2003). Gender, victimization, and outcomes: Reconceptualizing risk. Journal of Consulting and Clinical Psychology, 71, 528-539.

Pollard, M. W., Anderson, R. A., Anderson, W. T., \& Jennings, G. (1998). The development of a family forgiveness scale. Journal of Family Therapy, 20(1), 95-109. doi:10.1111/1467- 6427.00070

Raskin, R., \& Terry, H. (1988). A principal-components analysis of the Narcissistic Personality Inventory and further evidence of its construct validity. Journal of Personality and Social Psychology, 54, 890-902.

Rosenberg, M., (1979). Conceiving the Self. New York: Basic Books.ischp:

Rosenberg, M. (1965a). Society and the adolescent child. Princeton, NJ: Princeton University Press.

Rosenberg, M. (1965b). Rosenberg self-esteem scale (RSE). Acceptance and commitment therapy. Measures package, 61, 52.

Ruini, C. (2017). Positive psychology in the clinical domains: Research and practice. Springer.

Rusbult, C. E., Martz, J. M., \& Agnew, C. R. (1998). The investment model scale: Measuring commitment level, satisfaction level, quality of alternatives, and investment size. Personal Relationships, 5, 357-391.

Sackett, L. A., \& Saunders, D. G. (1999). The impact of different forms of psychological abuse on battered women. Violence and Victims, 14(1), 105-117. 
Sansone, R. A., Chu, J., \& Wiederman, M. W. (2007). Suicide attempts and domestic violence among women psychiatric inpatients. International Journal of Psychiatry in Clinical Practice, 11(2), 163166.

Scheier, M. F., \& Carver, C. S. (1985). Optimism, coping, and health: Assessment and implications of generalized outcome expectancies. Health Psychology, 4, 219-247.

Seligman, M. P., \& Csikszentmihalyi, M. (2000). Positive Psychology: An introduction. American Psychologist, 55(1), 5-14.

Sharot, T. (2011). The optimism bias: A tour of the irrationally positive brain. New York, NY, US: Pantheon/Random House.

Sharot, T., Korn, C. W., \& Dolan, R. J. (2011). How unrealistic optimism is maintained in the face of reality. Nature neuroscience, 14(11), 1475.

Sherin, K. M., Sinacore, J. M., Li, X., Zitter, R. E., \& Shakil, A. (1998). HITS: A short domestic violence screening tool for use in a family practice setting. Family Medicine, 30, 508-512.

Sin, N. L., \& Lyubomirsky, S. (2009). Enhancing well-being and alleviating depressive symptoms with positive psychology interventions: A practice-friendly meta-analysis. Journal of clinical psychology, 65(5), 467-487.

Snyder, C. R., Harris, C., Anderson, J. R., Holleran, S. A., Irving, L. M., Sigmon, S. T., et al. (1991). The will and the ways: Development and validation of an individual-differences measure of hope. Journal of Personality and Social Psychology, 60, 570-585.

Speckhard, A., Tarabrina, N., Krasnov, V., \& Mufel, N. (2005). Stockholm Effects and Psychological Responses to Captivity in Hostages Held by Suicide Terrorists. Traumatology, 11(2), 121-140.

Sprecher, S., \& Fehr, B. (2005). Compassionate love for close others and humanity. Journal of Social and Personal Relationships, 22, 629-652.

Stanley, S. M., \& Markman, H. J. (1992). Assessing commitment in personal relationships. Journal of Marriage and the Family, 54, 595-608.

Straus, M. A. (1979). Measuring intrafamily conflict and violence: The Conflict Tactics (CT) scales. Journal of Marriage and Family, 41, 75-88.

Strickland, P., \& Allen, G. (2017). Domestic Violence in England and Wales. House of Commons Library briefing paper 6377, London: House of Commons.

Sullivan, C. M., Tan, C., Basta, J., Rumptz, M., \& Davidson, W. (1992). An advocacy intervention program for women with abusive partners: Initial evaluation. American Journal of Community Psychology, 20, 309-332.

Tsang, J., \& Stanford, M. S. (2007). Forgiveness for intimate partner violence: The influence of victim and offender variables. Personality and Individual Differences, 42(4), 653-664.

Women, U. N. (2014). Facts and figures: Ending violence against women. United Nations Entity for Gender Equality and the Empowerment of Women.

Valdez, C. E., \& Lilly, M. M. (2015). Posttraumatic growth in survivors of intimate partner violence: An assumptive world process. Journal of Interpersonal Violence, 30(2), 215-231.

Varvaro, F., \& Palmer, M. (1993). Promotion of adaptation in battered women: A self-efficacy approach. Journal of the American Academy of Nurse Practitioners, 5, 264-270.

Vaux, A., Riedel, S., \& Stewart, D. (1987). Modes of social sup- port: The Social Support Behaviors Scale. American Journal of Community Psychology, 15, 209-237. 
Vitaliano, P. P. (1985). Manual for Appraisal Dimension Scale and revised ways of coping check-list. Seattle: Department of Psychiatry, University of Washington.

Walker, L. E. (2016). The battered woman syndrome. Springer publishing company.

Wendt, S. (2016). Domestic violence and feminism. In S. Wendt \& N. Moulding (Eds.), Contemporary feminisms in social work practice (pp. 209-219). New York, NY, US: Routledge/Taylor \& Francis Group.

Weinstein, N. (1980). Unrealistic optimism about future life events. Journal of Personality and Social Psychology, 39, 806-460.

Woods, S. J. (2000). Prevalence and patterns of posttraumatic stress disorder in abused and postabused women. Issues in Mental Health Nursing, 21, 309-324.

Zhang, Y., Zou, S., Cao, Y., \& Zhang, Y. (2012). Relationship between domestic violence and postnatal depression among pregnant Chinese women. International Journal of Gynecology $\mathcal{E}$ Obstetrics, 116(1), 26-30. 